\title{
Surgical Management of Necrotic Wounds Following Rituximab Therapy for Waldenstrom's Macroglobulinemia: A Case Report
}

Kyle Lockart, M.A., Ernesto Quinto, Jr., M.D*., Laurence Ferber, M.D.,

College of Medicine, University of Central Florida, United States.

Article Details
Article Type: Case Report
Received date: $31^{\text {st }}$ October, 2020
Accepted date: $17^{\text {th }}$ December, 2020
Published date: $21^{\text {st }}$ December, 2020
*t. Corresponding Author: Ernesto Quinto, Jr., M.D., College of Medicine, University of Central Florida/HCA GME Consor-
tium, United States. E-mail: Equinto21@gmail.com
Citation: Lockart, K., Quinto, E., \& Ferber, L. (2020). Surgical Management of Necrotic Wounds Following Rituximab Ther-
apy for Waldenstrom's Macroglobulinemia: A Case Report. J Case Reports Cancer 1(1):105. doi: https://doi.org/10.33790/
jcrc1100105.
Copyright: O2020, This is an open-access article distributed under the terms of the Creative Commons Attribution License
4.0, which permits unrestricted use, distribution, and reproduction in any medium, provided the original author and source are
credited.

\section{Abstract}

We report the case of a 77-year-old woman who developed extensive necrosis of the skin and subcutaneous tissue of bilateral lower extremities following administration of Rituximab antibody therapy for Waldenstrom's macroglobulinemia. The morphology and presentation of these lesions may represent calciphylaxis, a rare lifethreatening disease characterized by soft tissue necrosis. We wish to inform the medical community of the potential for developing this rare condition in patients, especially those with concomitant risk factors, undergoing antibody therapy interventions.

Keywords: Waldenstrom's Macroglobulinemia, Non Hodgkin Lymphoma, Pediatric Ovarian Cancer, Skin Necrosis, Calciphylaxis, Monoclonal Antibody Therapy

\section{Introduction}

Management of chronic, soft tissue wounds poses a complex challenge for the medical community. These lesions often present similarly with highly varied etiology. Previous studies have demonstrated the development of wounds such as non-healing ulcers, drug-induced necrosis, and calciphylaxis following pharmaceutical treatment. Calciphylaxis is a rare disorder affecting the skin and subcutaneous tissues presenting with painful and immobilizing soft tissue wounds. It is traditionally seen among patients with end stage kidney disease (ESKD) or renal transplants, but there has been association with nonuremic calciphylaxis among those with malignancy and tumor lysis syndrome following anticancer therapeutics including monoclonal antibody therapy [2-6]. The pathogenesis of this disease is poorly understood, but involves calcification and thrombosis of small vessels leading to infarcts in the dermis and subcutaneous adipose tissue. This presents clinically as livedo racemosa before progression to non-healing ulcers and eschars. This may appear as a confluent wound or as a network of smaller lesions with bridging areas of cutaneous discoloration $[1,7]$. The lesions often occur in the lower extremity as well as the abdomen or other areas of consolidated adipose tissue and are frequently bilateral. Severe, often debilitating pain is the most prevalent manifestation among patients clinically and is often out of proportion to the evident wounds [1]. Many patients afflicted are no longer able to mobilize due to pain and require the use of a wheelchair or hospitalization [8]. Chronic renal failure, female sex, obesity, diabetes, hypercoagulability, Vitamin $\mathrm{K}$ deficiency, and alterations in calcium and phosphate homeostasis have been demonstrated as risk factors for this condition. Our objective is to increase the surveillance of this rare condition in patients undergoing treatment with anticancer agents that are known to cause tumor lysis syndrome causing altered mineral homeostasis and predisposing patients to calciphylaxis. Additionally, we would like to inform the medical community of potentiators of this condition to improve early diagnosis and therapy.

\section{Case}

This case involves a 77-year-old woman with a history of Waldenstrom's macroglobulinemia, chronic kidney disease, cirrhosis, pediatric ovarian cancer, previous myocardial infarction, and hypertension who presented to the hospital for inability to ambulate due to a nine-month history of non-healing wounds on bilateral lower extremities. The patient reports these lesions appeared three to four weeks after starting Rituximab therapy which began one month following the diagnosis of Waldenstrom's macroglobulinemia. She described an initial reaction to the Rituximab infusion including feeling feverish with shaking throughout her body and a feeling of impending doom. The wounds began on her bilateral feet as painful, ecchymotic lesions that radiated cephalad until reaching her thighs. However, despite termination of therapy, the wounds continued to worsen and progressed to include central necrotic lesions with violaceous and ecchymotic surroundings (Figure 1). Initially, there was greater involvement on the left thigh over the right thigh. The oncologist recommended continuing treatment for her hematologic cancer with Ibrutinib, however, she refused further treatment. She underwent computed tomography of the area which demonstrated moderate subcutaneous and intramuscular edema with no acute osseous abnormality. The patient promptly sought treatment for these wounds by her dermatologist for which she received a topical antibiotic with minimal relief. Continued progression of these wounds resulting in pain causing difficulty with ambulation prompted admission to our hospital and further surgical consultation.

On admission, the patient presented with tender ecchymotic areas on the left thigh. Muscle tone and strength did not appear diminished and full range of motion was retained. The patient experienced pain at the site of eschar with flexion at the left hip against resistance. Review of her home meds demonstrated metoprolol, alprazolam, clonidine, furosemide, spironolactone, lisinopril, folic acid, pravastatin, allopurinol and cholecalciferol. She had recently terminated use of Warfarin due to frequent epistaxis. 

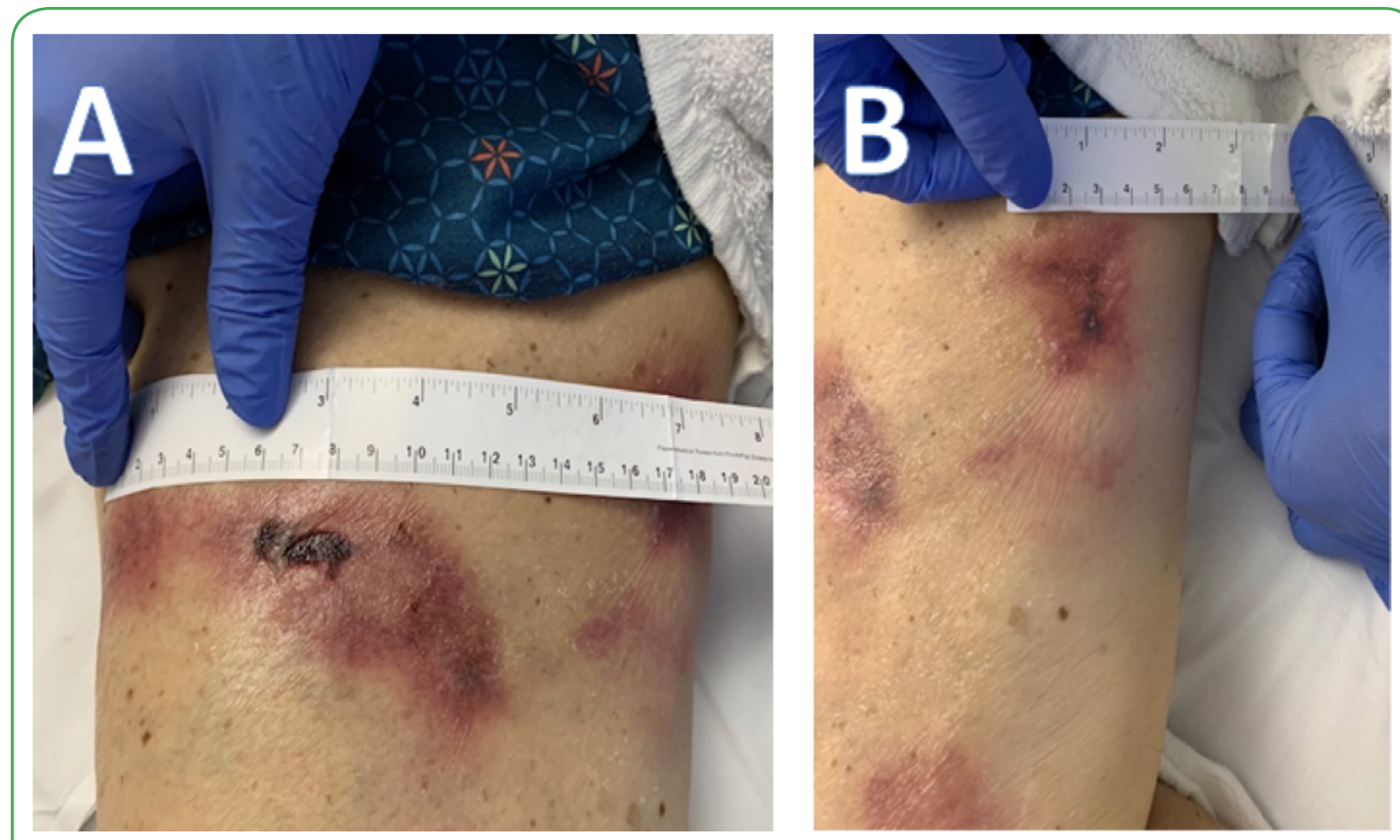

Figure 1: Gross images of necrotic, lower extremity wounds. Image A found on lateral aspect of left thigh. Image B found on medial aspect of right thigh.
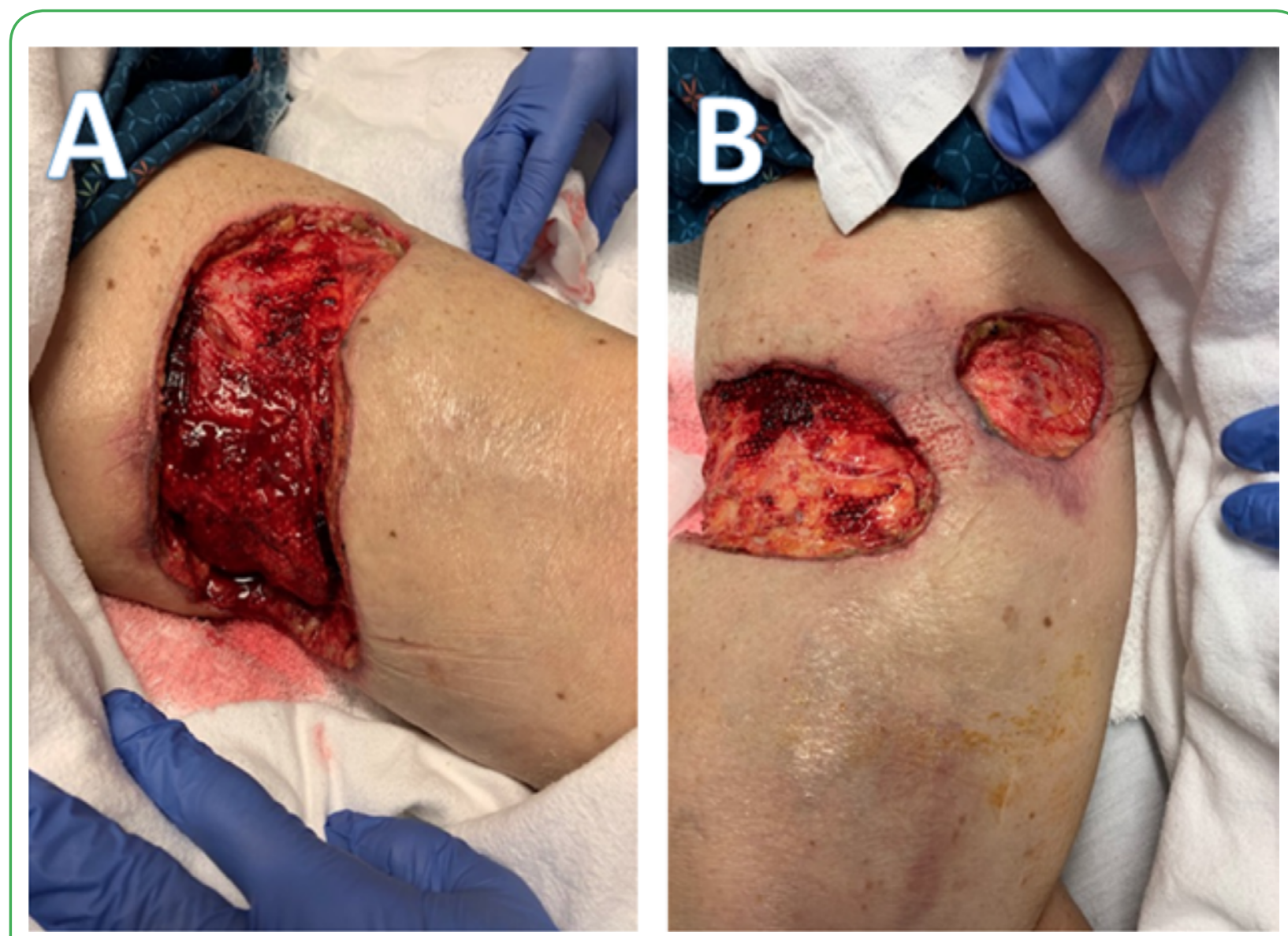

Figure 2: Gross images of lower extremity wounds following final excisional debridement procedure. Image A found on left thigh. Image B found on anterior and medial aspects of right thigh. 
Due to progression of the wounds despite medical treatment from her dermatologist, surgical debridement was deemed necessary. The patient provided written consent for surgery and underwent excisional debridement of eschar, skin, and partial-thickness subcutaneous tissue of the left thigh to final dimensions $13.5 \times 5.5 \times 0.5 \mathrm{~cm}$ with placement of a wound vac. The excised tissue was sent for surgical pathology and histologic appearance of the specimen demonstrated extensive skin necrosis with necrotic subcutaneous tissue but was overall negative for malignancy. Over the course of the next two days, two necrotic wounds similar to that of the left thigh developed on the right thigh. In addition, upon dressing removal of the left thigh wound, the remaining subcutaneous tissue appeared devascularized and necrotic. The patient was taken to surgery the next day for a second debridement of the left thigh wound to final dimensions 16 $\mathrm{x} 6 \times 0.5 \mathrm{~cm}$ left thigh and debridement of the right thigh wounds to final dimensions $13 \times 3.5 \times 0.5 \mathrm{~cm}$ right anterior thigh wound and $5 \times 4 \times 0.5 \mathrm{~cm}$ right medial thigh wound with application of wound vacs to all three sites. Once again, the patient's wounds failed to improve over the course of the next three days. She was taken to the operating room for the third and final procedure including excisional debridement of eschar, skin, and subcutaneous tissue down to fascia to final dimensions $17 \times 8 \times 2 \mathrm{~cm}$ left thigh wound, $15 \times 7 \times 2$ right anterior thigh wound, and $6 \times 5 \times 2 \mathrm{~cm}$ right medial thigh wound (Figure 2). The surgical specimens were sent for gram stain and culture which showed no evidence of bacterial growth.

Following her last surgical procedure, the patient remained in the hospital for treatment and observation of acute kidney injury superimposed on chronic kidney disease. Creatinine levels were found to be 2.7 upon admission and diminished to 1.3 at the end of her thirteen day admission following intravenous fluid administration and restriction of nephrotoxic medications including lisinopril, furosemide and spironolactone. Serum levels of calcium and phosphate were within normal reference ranges throughout her admission. Of note, epoetin alpha was prescribed and administered on hospital day 5 for anemia. She was discharged to home with wound vac care and instructions to return to clinic for follow-up.

23 days following discharge, the patient was readmitted to the inpatient medical service for complaints of nausea and vomiting and was found to be anemic secondary to a GI bleed. On day 2 of this admission, the patient was found to have Clostridium difficile colitis. Again, she was found to have creatinine levels between 3.8 - 4.5 during this time but refused further treatment. Per the patient's request, she was discharged to hospice care on hospital day 3 . We were informed of the patient's death the following day.

\section{Discussion}

Soft tissue wounds pose a complex challenge to treating physicians. Our case introduces that of a rare, chronic wound possibly resembling calciphylaxis. A review of the literature demonstrated malignancy as one of the most common causes of non-uremic calciphylaxis making up $22 \%$ of the 36 reported cases. Additionally, a number of case reports exhibited a connection between necrotic wounds and anticancer agents including monoclonal antibody therapy [9-11]. Although Rituximab was not specifically listed among the agents utilized in treatment of all patients, this drug and others reported are associated with tumor lysis syndrome which may result in hyperuricemia and hyperphosphatemia. This disturbance in mineral homeostasis may influence vascular calcium chelation leading to calciphylaxis [12]. One previous report similarly illustrated development of a non-specific necrotic wound following Rituximab therapy for Waldenstrom's Macroglobulinemia. The patient is described as having a reaction to treatment as well consisting of rigors, oxygen desaturation, and a febrile nonhemolytic reaction. Following treatment, ischemic changes were noted on the patient's nose and laboratory testing demonstrated findings of disseminated intravascular coagulation and acute renal failure. The patient's nose was found to have tender, violaceous skin on the nose that progressed to a necrotic eschar over the following week [10].

Due to the complex medical history of the patient reported, it is difficult to determine the true etiology of her necrotic wounds. Her previous diagnoses are consistent with many of the risk factors listed above. Chronic kidney disease may have predisposed her to the traditional calcific uremic arteriopathy, however this is typically accompanied by elevated levels of serum calcium and phosphate levels that were not represented during her admission [1]. In addition, renal function did not reach the threshold for the need of hemodialysis. The use of Warfarin may have promoted vascular calcification by way of disinhibiting the vitamin K-dependent matrix Gla protein the prevents calcium deposition in arteries [13]. Additionally the use of cholecalciferol mechanistically elevates serum calcium levels which may also promote vascular deposition [1]. The patient has consistently taken both of these medications for a prolonged time without complication. Finally, the diagnosis of Waldenstrom's Macroglobulinemia has the potential to increase coagulability due to hyperviscosity syndrome secondary to elevated lymphoplasmacytic cells, increasing the risk of vascular thrombosis [14]. Any number of these individually could have resulted in the onset of these wounds, but it is the chronological association with her anticancer therapy and subjective complaints that raise concern for the potential for an adverse effect of Rituximab. The pathology report provided for the surgical specimen following debridement did not describe the vascular calcifications that would demonstrate a clear diagnosis for calciphylaxis. However, the literature states that a diagnostic biopsy should be taken from the margin of an active lesion rather than an area of central necrosis such as that provided after the procedure. Additionally, diagnosis can be made without biopsy in patients with chronic kidney disease with a classic presentation of painful necrotic ulcers and black eschars [1, 15].

The treatment determined most appropriate for our patient's presentation was surgical debridement to remove the necrotic area and allow for regeneration of healthy tissue. Previous studies agree that this is a favorable treatment for those diagnosed with calciphylaxis and correlated with prolonged survival $[8,16]$. Those who are not good candidates for surgery or who will not consent for a surgical procedure may benefit from intravenous sodium thiosulfate therapy [17]. The prognosis remains poor despite treatment for those diagnosed with calciphylaxis caused by uremic and nonuremic modalities alike [1]. Additionally, lack of awareness regarding this condition may result in under recognition and under reporting limiting the catalogue of available resources [18]. Heightened surveillance of this entity from a multidisciplinary cohort of dermatologists, nephrologists, surgeons, and primary care physicians may allow for earlier diagnosis and favorable response to treatment.

\section{Conclusion}

Calciphylaxis is a rare condition associated with significant morbidity and mortality and should be considered in patients with necrotic wounds and pain out of proportion to physical findings. Heightened clinical suspicion for this disease should be considered in those with concomitant risk factors including renal disease, malignancy, and use of pharmaceutical agents that may increase susceptibility to vascular calcification. We present a case with clinical presentation consistent with calciphylaxis following administration of Rituximab therapy for Waldenstrom's macroglobulinemia in an effort to alert the medical community of a potential association of the anticancer agent and this disease. In addition, we hope to alert the medical community of this under reported diagnosis and associated risk factors to increase surveillance of the disease.

Statement of Informed Consent: The presented patient provided informed consent to the use of the data concerning their case for use in this publication. 
Disclaimer: This research was supported (in whole or in part) by HCA Healthcare and/or an HCA Healthcare affiliated entity. The views expressed in this publication represent those of the author(s) and do not necessarily represent the official views of HCA Healthcare or any of its affiliated entities.

\section{Acknowledgements: None}

Competing interests: The author(s) declare that they have no competing interests.

\section{References}

1. Nigwekar, S.U. (2017). Calciphylaxis. Current opinion in nephrology and hypertension,26(4), 276-81.

2. Morand, M., Chababi-Atallah, M., Jean, S.É., \& Fernandes, C. (2018). Calciphylaxis in a Patient With Non-Hodgkin Lymphoma: Case Report and Literature Review. Journal of cutaneous medicine and surgery, 22(5), 524-6.

3. Bosler, D.S., Amin, M.B., Gulli, F., \& Malhotra, R.K. (2007). Unusual case of calciphylaxis associated with metastatic breast carcinoma. The American Journal of dermatopathology, 29(4), 400-3.

4. Goff, H.W., \& Grimwood, R.E. (2005). A case of calciphylaxis and chronic myelomonocytic leukemia. Cutis, 75(6), 325-8.

5. Golitz, L.E., \& Field, J.P. (1972). Metastatic calcification with skin necrosis. Archives of dermatology, 106(3), 398-402.

6. Yang, H., Rosove, M.H., \& Figlin, R.A. (1999). Tumor lysis syndrome occurring after the administration of rituximab in lymphoproliferative disorders: high-grade non-Hodgkin's lymphoma and chronic lymphocytic leukemia. American journal of hematology, 62(4), 247-50.

7. Budisavljevic, M.N., Cheek, D., \& Ploth, D.W. (1996). Calciphylaxis in chronic renal failure. Journal of the American Society of Nephrology : JASN, 7(7), 978-82.

8. Weenig, R.H., Sewell, L.D., Davis, M.D., McCarthy, J.T., \& Pittelkow, M.R. (2007). Calciphylaxis: natural history, risk factor analysis, and outcome. Journal of the American Academy of Dermatology, 56(4), 569-79.

9. Goyal, S., Huhn, K.M., \& Provost, T.T. (2000). Calciphylaxis in a patient without renal failure or elevated parathyroid hormone: possible aetiological role of chemotherapy. The British journal of dermatology, 143(5), 1087-90.
10. Pearlman, A.N., Fechner, F.P., \& Constantinides, M. (2006). Development of nasal skin necrosis associated with rituximab treatment for Waldenström's macroglobulinemia and subsequent spontaneous resolution. Ear, nose, \& throat journal, 85(7), 4313.

11. Botden, I.P., Leys, M.B., van Houten, A.A., \& Peeters, R.P. (2008). Severe skin necrosis after rituximab-CHOP therapy. The Netherlands journal of medicine, 66(10), 448-9.

12. Wilson, F.P., \& Berns, J.S. (2014). Tumor lysis syndrome: new challenges and recent advances. Advances in chronic kidney disease, 21(1), 18-26.

13. Schurgers, L.J., Cranenburg, E.C., \& Vermeer, C. (2008). Matrix Gla-protein: the calcification inhibitor in need of vitamin K. Thrombosis and haemostasis, 100(4), 593-603.

14. Jalali, S., \& Ansell, S.M.(2016). Bone marrow microenvironment in Waldenstrom's Macroglobulinemia. Best practice \& research. Clinical haematology, 29(2), 148-55.

15. Jiménez-Gallo, D., Ossorio-García, L., \& Linares-Barrios, M. (2015). Calcinosis Cutis and Calciphylaxis. Actas dermosifiliograficas, 106(10), 785-94.

16. McCarthy, J.T., El-Azhary, R.A., Patzelt, M.T., Weaver, A.L., Albright, R.C., Bridges, A.D., ... \& Williams, A.W. (2016). Survival, Risk Factors, and Effect of Treatment in 101 Patients With Calciphylaxis. Mayo Clinic proceedings, 91(10), 1384-94.

17. Nigwekar, S.U., Brunelli, S.M., Meade, D., Wang, W., Hymes, J., \& Lacson, E. (2013). Sodium thiosulfate therapy for calcific uremic arteriolopathy. Clinical journal of the American Society of Nephrology : CJASN, 8(7), 1162-70.

18. Nigwekar, S.U., Wolf, M., Sterns, R.H., \& Hix, J.K. (2008). Calciphylaxis from nonuremic causes: a systematic review. Clinical journal of the American Society of Nephrology : CJASN, 3(4), 1139-43. 